\title{
Fixed point result for rational type $\varphi$-Geraghty contraction
}

\author{
ÖZLEM ACAR
}

\begin{abstract}
In this paper, we introduce the notions of rational type Geraghty contractions. Using this type of contraction, we investigate under which conditions such mappings posses a unique fixed point in the framework of complete metric spaces.
\end{abstract}

\section{INTRODUCTION AND PRELIMINARIES}

Fixed point theory on a metric space is started by Banach in 1922. Banach's Contraction Principle says that, whenever $(M, \sigma)$ is complete, then any contraction selfmap of $M$ has a unique fixed point. Afterwards, the crucial role of the principle in existence and uniqueness problems arising in mathematics has been realized which fact directed the researchers to extend and generalize the principle in many ways, see e.g. $[1,2,4-7,9,10]$. No doubtly, one of those is given by Geraghty [8] such that:

Let $S$ be the family of all functions $\beta:[0, \infty) \rightarrow[0,1)$ which satisfies the condition

$$
\lim _{n \rightarrow \infty} \beta\left(t_{n}\right)=1 \Longrightarrow \lim _{n \rightarrow \infty} t_{n}=0 .
$$

Theorem 1. Let $(M, \sigma)$ be a complete metric space and $T: M \rightarrow M$ be a mapping. Suppose that there exists $\beta \in S$ such that

$$
\sigma(T a, T b) \leq \beta((\sigma(a, b)) \sigma(a, b),
$$

for all $a, b \in M$. Then $T$ has a unique fixed point $z \in M$. Moreover, for any initial point $a_{0} \in M$, the iterative sequence $\left\{T^{n} a_{0}\right\}_{n=1}^{\infty}$ converges to $z$.

Recently, in [3] the authors proved the following fixed point theorems that were inspired from the well-known results of Suzuki [10].

Definition $1([3])$. Suppose that $\varphi:[0, \infty) \rightarrow[0, \infty)$ is a function and $\beta \in S$. A self mapping $T$ on a complete metric space $(M, \sigma)$ is called $\varphi$-Geraghty contraction if it satisfies the following conditions:

2020 Mathematics Subject Classification. Primary: 54H25 Secondary: 47H10.

Key words and phrases. Fixed point, Geraghty contractions, rational type.

Full paper. Received 10 February 2021, revised 9 March 2021, accepted 28 March 2021, available online 15 June 2021. 
$\left(\varphi_{1}\right) \varphi(t)<t$, for all $t \in(0, \infty)$.

$\left(\varphi_{2}\right)$ For any $\epsilon>0$, there exists a $\delta>0$ such that

$$
\epsilon<t<\epsilon+\delta \text { implies } \varphi(t) \leq \epsilon .
$$

$\left(\varphi_{3}\right) \sigma(T a, T b) \leq \beta((\sigma(a, b))(\varphi \circ \sigma(a, b))$, for all $a, b \in M$.

Theorem $2([3])$. Let $(M, \sigma)$ be a complete metric space. If a self mapping $T: M \rightarrow M$ forms a $\varphi$-Geraghty contraction, then $T$ has a unique fixed point $z \in M$. Moreover, $\left\{T^{n} a\right\}$ converges to $z$ for all $a \in M$.

Let $S^{\prime}$ be the family of all functions $\beta:[0, \infty) \rightarrow[0,1)$ which satisfies the condition

$$
\limsup _{n \rightarrow \infty} \beta\left(t_{n}\right)=1 \Longrightarrow \lim _{n \rightarrow \infty} t_{n}=0 .
$$

Definition 2 ([3]). Suppose that $\varphi:[0, \infty) \rightarrow[0, \infty)$ is a function and $\beta \in S^{\prime}$. A self mapping $T$ on a complete metric space $(M, \sigma)$ is called Ćirić type $\varphi$-Geraghty contraction if it satisfies the following conditions:

$\left(\varphi_{0}\right) \varphi$ is upper semicontinuous.

$\left(\varphi_{1}\right) \varphi(t)<t$, for all $t \in(0, \infty)$.

$\left(\varphi_{2}\right)$ For any $\epsilon>0$, there exists a $\delta>0$ such that

$$
\epsilon<t<\epsilon+\delta \text { implies } \varphi(t) \leq \epsilon .
$$

$\left(\varphi_{3}\right) \sigma(T a, T b) \leq \beta((L(a, b)(\varphi \circ L(a, b))$, where

$$
L(a, b)=\max \left\{\sigma(a, b), \frac{\sigma(a, T b)+\sigma(b, T a)}{2}, \sigma(a, T a), \sigma(b, T b)\right\} .
$$

Theorem 3 ([3]). Let $(M, \sigma)$ be a complete metric space. If a self mapping $T: M \rightarrow M$ forms a $\varphi$-Geraghty contraction, then $T$ has a fixed point $z \in M$. Moreover, $\left\{T^{n} a\right\}$ converges to $z$ for all $a \in M$.

Remark $1([3])$. By $\left(\varphi_{1}\right)$ it is easy tho see that $\varphi_{2}$ is equivalent to following: $\left(\varphi_{2}^{\prime}\right)$ For any $\varepsilon>0$ there exists $\delta>0$ such that $t<\varepsilon+\delta$ implies $\varphi(t) \leq \varepsilon$. Indeed, if $0<t \leq \varepsilon$, from $\left(\varphi_{1}\right)$ we have $\varphi(t)<t \leq \varepsilon$.

In this paper, we introduce rational type Geraghty contraction and prove some results for this type contraction.

\section{MAIN RESUlT}

Let $F^{\prime}$ be the family of all functions $\beta:[0, \infty) \rightarrow[0,1)$ which satisfies the condition

$$
\limsup _{n \rightarrow \infty} \beta\left(t_{n}\right)=1 \Longrightarrow \lim _{n \rightarrow \infty} t_{n}=0 .
$$

Definition 3. Suppose that $\varphi:[0, \infty) \rightarrow[0, \infty)$ is a function and $\beta \in F^{\prime}$. A self mapping $T$ on a complete metric space $(M, \sigma)$ is called rational type $\varphi$-Geraghty contraction if it satisfies the following conditions:

$\left(\varphi_{0}\right) \varphi$ is upper semicontinuous. 
$\left(\varphi_{1}\right) \varphi(t)<t$ for all $t \in(0, \infty)$.

$\left(\varphi_{2}\right)$ for any $\epsilon>0$, there exists a $\delta>0$ such that

$$
\epsilon<t<\epsilon+\delta \text { implies } \varphi(t) \leq \epsilon .
$$

$\left(\varphi_{3}^{\prime}\right) \sigma(T a, T b) \leq \beta(K(a, b))(\varphi(K(a, b)))$, where

$K(a, b)=\max \left\{\sigma(a, b), \sigma(a, f a), \sigma(b, f b), \frac{\sigma(a, f a) \sigma(b, f b)}{1+\sigma(a, b)}, \frac{\sigma(a, f a) \sigma(b, f b)}{1+\sigma(f a, f b)}\right\}$.

Theorem 4. Let $(M, \sigma)$ be a complete metric space. If a self mapping $T: M \rightarrow M$ forms a rational type $\varphi$-Geraghty contraction, then $T$ has a fixed point $w \in M$. Moreover, $\left\{T^{n} a\right\}$ converges to $w$ for all $a \in M$.

Proof. Let $a_{0} \in M$. We define a sequence $\left\{a_{n}\right\}$ in $M$ by $a_{n+1}=T a_{n}=$ $T^{n+1} a_{0}$, for all $n \geq 0$. Suppose that $\sigma\left(a_{n}, a_{n+1}\right)=0$ for some $n_{0}$. In this case, the proof is trivially completed. Consequently, we assume that

$$
\sigma\left(a_{n}, a_{n+1}\right) \neq 0,
$$

for all $n$. Thus we have $\sigma\left(a_{n}, a_{n+1}\right)>0$. By $\left(\varphi_{3}^{\prime}\right),\left(\varphi_{1}\right)$ and definition of function $\beta$, we have

$$
\begin{aligned}
& \sigma\left(a_{n+1}, a_{n+2}\right)=\sigma\left(T a_{n}, T a_{n+1}\right) \\
\leq & \beta\left(\max \left\{\begin{array}{l}
\sigma\left(a_{n}, a_{n+1}\right), \sigma\left(a_{n}, f a_{n}\right), \sigma\left(a_{n+1}, f a_{n+1}\right), \\
\frac{\sigma\left(a_{n}, f a_{n}\right) \sigma\left(a_{n+1}, f a_{n+1}\right)}{1+\sigma\left(a_{n}, a_{n+1}\right)}, \frac{\sigma\left(a_{n}, f a_{n}\right) \sigma\left(a_{n+1}, f a_{n+1}\right)}{1+\sigma\left(f a_{n}, f a_{n+1}\right)}
\end{array}\right\}\right) \\
& \times \varphi\left(\max \left\{\begin{array}{l}
\sigma\left(a_{n}, a_{n+1}\right), \sigma\left(a_{n}, f a_{n}\right), \sigma\left(a_{n+1}, f a_{n+1}\right), \\
\frac{\sigma\left(a_{n}, f a_{n}\right) \sigma\left(a_{n+1}, f a_{n+1}\right)}{1+\sigma\left(a_{n}, a_{n+1}\right)}, \frac{\sigma\left(a_{n}, f a_{n}\right) \sigma\left(a_{n+1}, f a_{n+1}\right)}{1+\sigma\left(f a_{n}, f a_{n}\right)}
\end{array}\right\}\right) \\
= & \beta\left(\max \left\{\begin{array}{l}
\sigma\left(a_{n}, a_{n+1}\right), \sigma\left(a_{n}, a_{n+1}\right), \sigma\left(a_{n+1}, a_{n+2}\right), \\
\frac{\sigma\left(a_{n}, a_{n+1}\right) \sigma\left(a_{n+1}, a_{n+2}\right)}{1+\sigma\left(a_{n}, a_{n+1}\right)}, \frac{\sigma\left(a_{n}, a_{n+1}\right) \sigma\left(a_{n+1}, a_{n+2}\right)}{1+\sigma\left(a_{n+1}, a_{n+2}\right)}
\end{array}\right\}\right) \\
< & \times \varphi\left(\max \left\{\begin{array}{l}
\sigma\left(a_{n}, a_{n+1}\right), \sigma\left(a_{n}, a_{n+1}\right), \sigma\left(a_{n+1}, a_{n+2}\right), \\
\frac{\sigma\left(a_{n}, a_{n+1}\right) \sigma\left(a_{n+1}, a_{n+2}\right)}{1+\sigma\left(a_{n}, a_{n+1}\right)}, \frac{\sigma\left(a_{n}, a_{n+1}\right) \sigma\left(a_{n+1}, a_{n+2}\right)}{1+\sigma\left(a_{n+1}, a_{n+2}\right)}
\end{array}\right\}\right) \\
< & \times\left(\max \left\{\sigma\left(a_{n}, a_{n+1}\right), \sigma\left(a_{n+1}, a_{n+2}\right)\right\}\right) \\
< & \varphi\left(\sigma\left(a_{n}, a_{n+1}\right)\right) \\
< & \sigma\left(a_{n}, a_{n+1}\right),
\end{aligned}
$$

for all $n \geq 0$. Hence the non-negative real number sequence $\left\{\sigma\left(a_{n}, a_{n+1}\right)\right\}$ is non-increasing. Consequently, this sequence convergence to some $\epsilon>0$. 
We claim that $\epsilon=0$. Firstly, we note $\epsilon<\sigma\left(a_{n}, a_{n+1}\right)$ for all $n \geq 0$. Arguing by contradiction, we assume $\epsilon>0$. Then by $\left(\varphi_{2}^{\prime}\right)$ from Remark 1 , there exists $\delta>0$ such that

$$
t<\epsilon+\delta \text { implies } \varphi(t) \leq \epsilon .
$$

On the other hand, for sufficiently large $N \in \mathbb{N}$ we have

$$
0<\epsilon<\sigma\left(a_{N}, a_{N+1}\right)<\epsilon+\delta,
$$

and, taking into account the property $\left(\varphi_{2}^{\prime}\right)$, we get

$$
\begin{aligned}
& 0<\epsilon \leq \sigma\left(a_{N+2}, a_{N+3}\right)<\sigma\left(a_{N+1}, a_{N+2}\right) \\
& =\sigma\left(T a_{N}, T a_{N+1}\right) \\
& \leq \beta\left(K\left(a_{N}, a_{N+1}\right)\right)\left(\varphi\left(K\left(a_{N}, a_{N+1}\right)\right)\right) \\
& \leq \beta\left(\max \left\{\begin{array}{l}
\sigma\left(a_{N}, a_{N+1}\right), \sigma\left(a_{N}, f a_{N}\right), \sigma\left(a_{N+1}, f a_{N+1}\right), \\
\frac{\sigma\left(a_{N}, f a_{N}\right) \sigma\left(a_{N+1}, f a_{N+1}\right)}{1+\sigma\left(a_{N}, a_{N+1}\right)}, \frac{\sigma\left(a_{N}, f a_{N}\right) \sigma\left(a_{N+1}, f a_{N+1}\right)}{1+\sigma\left(f a_{N}, f a_{N+1}\right)}
\end{array}\right\}\right) \\
& \times\left(\varphi\left(\max \left\{\begin{array}{l}
\sigma\left(a_{N}, a_{N+1}\right), \sigma\left(a_{N}, f a_{N}\right), \sigma\left(a_{N+1}, f a_{N+1}\right), \\
\frac{\sigma\left(a_{N}, f a_{N}\right) \sigma\left(a_{N+1}, f a_{N+1}\right)}{1+\sigma\left(a_{N}, a_{N+1}\right)}, \frac{\sigma\left(a_{N}, f a_{N}\right) \sigma\left(a_{N+1}, f a_{N+1}\right)}{1+\sigma\left(f a_{N}, f a_{N+1}\right)}
\end{array}\right\}\right)\right) \\
& =\beta\left(\max \left\{\begin{array}{l}
\sigma\left(a_{N}, a_{N+1}\right), \sigma\left(a_{N}, a_{N+1}\right), \sigma\left(a_{N+1}, a_{N+2}\right), \\
\frac{\sigma\left(a_{N}, a_{N+1}\right) \sigma\left(a_{N+1}, a_{N+2}\right)}{1+\sigma\left(a_{N}, a_{N+1}\right)}, \frac{\sigma\left(a_{N}, a_{N+1}\right) \sigma\left(a_{N+1}, a_{N+2}\right)}{1+\sigma\left(a_{N+1}, a_{N+2}\right)}
\end{array}\right\}\right) \\
& \times\left(\varphi\left(\max \left\{\begin{array}{l}
\sigma\left(a_{N}, a_{N+1}\right), \sigma\left(a_{N}, a_{N+1}\right), \sigma\left(a_{N+1}, a_{N+2}\right), \\
\frac{\sigma\left(a_{N}, a_{N+1}\right) \sigma\left(a_{N+1}, a_{N+2}\right)}{1+\sigma\left(a_{N}, a_{N+1}\right)}, \frac{\sigma\left(a_{N}, a_{N+1}\right) \sigma\left(a_{N+1}, a_{N+2}\right)}{1+\sigma\left(a_{N+1}, a_{N+2}\right)}
\end{array}\right\}\right)\right) \\
& \leq \beta\left(\max \left\{\sigma\left(a_{N}, a_{N+1}\right), \sigma\left(a_{N+1}, a_{N+2}\right)\right\}\right) \\
& \times\left(\varphi\left(\max \left\{\sigma\left(a_{N}, a_{N+1}, \sigma\left(a_{N+1}, a_{N+2}\right)\right\}\right)\right)\right. \\
& \leq \varphi\left(\max \left\{\sigma\left(a_{N}, a_{N+1}, \sigma\left(a_{N+1}, a_{N+2}\right)\right\}\right)\right. \\
& <\sigma\left(a_{N}, a_{N+1}\right) \leq \epsilon,
\end{aligned}
$$

a contradiction. Thus we have

$$
\lim _{n \rightarrow \infty} \sigma\left(a_{n}, a_{n+1}\right)=0 .
$$

Now we show that $\left\{a_{n}\right\}$ is a Cauchy sequence. Let $\epsilon_{1}>0$ fixed. Then there exists $\delta_{1}>0$ which satisfies the following

$$
t<\max \left\{\epsilon_{1}+\delta_{1}, \delta_{1}^{2}\right\} \Longrightarrow \varphi(t) \leq \epsilon_{1} .
$$


From (1) we can choose $k \in \mathbb{N}$ large enough to satisfy $\sigma\left(a_{k}, a_{k+1}\right)<\delta_{1}(\epsilon)<$ $\delta_{1}$. We will show by induction that

$$
\sigma\left(a_{k}, a_{k+l}\right)<\epsilon_{1}+\delta_{1}
$$

for all $k \in \mathbb{N}$. We have already proved for $k=1$, so we suppose the condition (3) is satisfied for some $j \in \mathbb{N}$. For $l=j+1$, we get

$$
\begin{aligned}
K\left(a_{k}, a_{k+j}\right) & =\max \left\{\begin{array}{c}
\sigma\left(a_{k}, a_{k+j}\right), \sigma\left(a_{k}, f a_{k}\right), \sigma\left(a_{k+j}, f a_{k+j}\right), \\
\frac{\sigma\left(a_{k}, f a_{k}\right) \sigma\left(a_{k+j}, f a_{k+j}\right)}{1+\sigma\left(a_{k}, a_{k+j}\right)}, \frac{\sigma\left(a_{k}, f a_{k}\right) \sigma\left(a_{k+j}, f a_{k+j}\right)}{1+\sigma\left(f a_{k}, f a_{k+j}\right)}
\end{array}\right\} \\
& =\max \left\{\begin{array}{l}
\sigma\left(a_{k}, a_{k+j}\right), \sigma\left(a_{k}, a_{k+1}\right), \sigma\left(a_{k+j}, a_{k+j+1}\right), \\
\frac{\sigma\left(a_{k}, a_{k+1}\right) \sigma\left(a_{k+j}, a_{k+j+1}\right)}{1+\sigma\left(a_{k}, a_{k+j}\right)}, \frac{\sigma\left(a_{k}, a_{k+1}\right) \sigma\left(a_{k+j}, a_{k+j+1}\right)}{1+\sigma\left(a_{k+1}, a_{k+j+1}\right)}
\end{array}\right\} \\
& \leq \max \left\{\delta_{1}, \epsilon_{1}+\delta_{1}, \delta_{1}, \delta_{1}^{2}, \delta_{1}^{2}\right\} \\
\leq & \max \left\{\epsilon_{1}+\delta_{1}, \delta_{1}^{2}\right\} .
\end{aligned}
$$

Because, by $\left(\varphi_{3}^{\prime}\right)$ and $(2)$ we obtain

$$
\begin{aligned}
\sigma\left(a_{k}, a_{k+j+1}\right) & \leq \sigma\left(a_{k}, a_{k+1}\right)+\sigma\left(a_{k+1}, a_{k+j+1}\right) \\
& =\sigma\left(a_{k}, a_{k+1}\right)+\sigma\left(T a_{k}, T a_{k+j}\right) \\
& \leq \sigma\left(a_{k}, a_{k+1}\right)+\beta\left(\left(\lambda\left(a_{k}, a_{k+j}\right)\right)\left(\varphi \circ \lambda\left(a_{k}, a_{k+j}\right)\right)\right. \\
& <\epsilon_{1}+\delta_{1} .
\end{aligned}
$$

Consequently, (3) holds for $l=j+1$. Hence $\sigma\left(a_{k}, a_{k+l}\right)<\epsilon_{1}$ for all $k \in \mathbb{N}$ and $l \leq 1$, which means $\lim _{n \rightarrow \infty} \sup _{m>n} \sigma\left(a_{n}, a_{m}\right)=0$. So the sequence $\left\{a_{n}\right\}$ is Cauchy. Since $(M, \sigma)$ is complete, there exists $w \in M$ such that $a_{n} \rightarrow w$ when $n \rightarrow \infty$.

Now, we will show that $T w=w$. Suppose on the contrary, that there exists $r>0$ such that $r=\sigma(w, T w)>0$. Note that due to the fact the sequence $\left\{a_{n}\right\}$ is convergent to $w$, we can choose $l \in \mathbb{N}$ such that $\sigma\left(w, a_{n}\right)<\frac{r}{2}$ for all $n \geq l$. So, for $n \geq l$ we have

$$
\begin{aligned}
K\left(a_{n}, w\right) & =\max \left\{\begin{array}{l}
\sigma\left(a_{n}, w\right), \sigma\left(a_{n}, f a_{n}\right), \sigma(w, f w), \\
\frac{\sigma\left(a_{n}, f a_{n}\right) \sigma(w, f w)}{1+\sigma\left(a_{n}, w\right)}, \frac{\sigma\left(a_{n}, f a_{n}\right) \sigma(w, f w)}{1+\sigma\left(f a_{n}, f w\right)}
\end{array}\right\} \\
& \leq \max \left\{\begin{array}{l}
\frac{r}{2}, \sigma\left(a_{n}, a_{n+1}\right), \sigma(w, f w), \\
\frac{\sigma\left(a_{n}, a_{n+1}\right) \sigma(w, f w)}{1+\sigma\left(a_{n}, w\right)}, \frac{\sigma\left(a_{n}, a_{n+1}\right) \sigma(w, f w)}{1+\sigma\left(a_{n+1}, f w\right)}
\end{array}\right\} \\
& \leq \max \left\{\begin{array}{l}
\frac{r}{2}, \sigma\left(a_{n}, a_{n+1}\right), r, \\
\frac{\sigma\left(a_{n}, a_{n+1}\right) \sigma(w, f w)}{1+\sigma\left(a_{n}, w\right)}, \frac{\sigma\left(a_{n}, a_{n+1}\right) \sigma(w, f w)}{1+\sigma\left(a_{n+1}, f w\right)}
\end{array}\right\}
\end{aligned}
$$


It yields that $\lim _{n \rightarrow \infty} \sup K\left(a_{n}, w\right)=r$. By the triangle inequality together with $\left(\varphi_{3}\right)$ we derive that

$$
\begin{aligned}
0 & <r=\sigma(w, T w) \leq \sigma\left(w, a_{n+1}\right)+\sigma\left(a_{n+1}, T w\right) \\
& \leq \sigma\left(w, a_{n+1}\right)+\beta\left(K\left(a_{n}, w\right)\right)\left(\varphi\left(K\left(a_{n}, w\right)\right)\right) .
\end{aligned}
$$

Letting $n \rightarrow \infty$ in the previous inequality, together with $\left(\varphi_{0}\right)$ and $\left(\varphi_{1}\right)$ we get

$$
\begin{aligned}
0 & <r=\sigma(w, T w) \\
& \leq \lim _{n \rightarrow \infty} \sup \left[\sigma\left(w, a_{n+1}\right)+\beta\left(K\left(a_{n}, w\right)\right)\left(\varphi\left(K\left(a_{n}, w\right)\right)\right)\right] \\
& =\lim _{n \rightarrow \infty} \sup \beta\left(K\left(a_{n}, w\right)\right) \lim _{n \rightarrow \infty} \sup \varphi\left(K\left(a_{n}, w\right)\right) \\
& \leq \varphi(r)<r .
\end{aligned}
$$

Thus $\lim _{n \rightarrow \infty} \sup \left[\beta\left(K\left(a_{n}, w\right)\right)\right]=1$. Since $\beta \in F^{\prime}$, we have

$$
\lim _{n \rightarrow \infty} \sup K\left(a_{n}, w\right)=0 .
$$

Accordingly, we have $\sigma(w, T w)=r=0$, that is $w$ is a fixed point of $T$. As a last step, we indicate that the limit point $w$ of the iterative sequence $\left\{a_{n}\right\}$ is unique. Suppose on the contrary, that $v$ is another fixed point of $T$, with $w \neq v$. It is clear that $K(w, v)=\sigma(w, v)$. Thus we have

$$
\begin{aligned}
0 & <\sigma(w, v)=\sigma(T w, T v) \\
& \leq \beta(K((w, v))(\varphi(K((w, v))) \\
& \leq \beta(\sigma(w, v))(\varphi(\sigma(w, v))) \\
& <\sigma(w, v),
\end{aligned}
$$

a contradiction. So fixed point of $T$ is unique.

Corollary 1. Let $(M, \sigma)$ be a complete metric space. If a self mapping $T: M \rightarrow M$ forms a $\varphi$-Geraghty contraction such that

$$
\sigma(T a, T b) \leq \beta(\max \{\sigma(a, b), \sigma(a, T a), \sigma(b, T b)\})(\varphi(\sigma(a, b))),
$$

for all $a, b \in M$, then $T$ has a fixed point $w \in M$. Moreover, $\left\{T^{n} a\right\}$ converges to $w$ for all $a \in M$.

Corollary 2. Let $(M, \sigma)$ be a complete metric space. If a self mapping $T: M \rightarrow M$ forms a $\varphi$-Geraghty contraction such that

$$
\sigma(T a, T b) \leq \beta(\sigma(a, b))(\varphi(\sigma(a, b))),
$$

for all $a, b \in M$, then $T$ has a fixed point $w \in M$. Moreover, $\left\{T^{n} a\right\}$ converges to $w$ for all $a \in M$. 


\title{
REFERENCES
}

[1] Ö. Acar, V. Berinde, I. Altun, Fixed point theorems for Ćirić-type strong almost contractions on partial metric spaces, Journal of Fixed Point Theory and Applications, 12 (2012), 247-259.

[2] Ö. Acar, I. Altun, A fixed point theorem for $\psi_{F}-$ Geraghty contraction on metric like space, Fasciculi Mathematici, 59 (2017), 5-12.

[3] B. Alqahtani, A. Fulga, E. Karapınar, On Ćirić Type $\varphi-$ Geraghty Contractions, Thai Journal of Mathematics, 17 (1) (2019), 205-216.

[4] E. Karapinar, A Short Survey on the Recent Fixed Point Results on b-Metric Spaces, Constructive Mathematical Analysis, 1 (1) (2018), 15-44.

[5] E. Karapinar, $\alpha-\psi-$ Geraghty contraction type mappings and some related fixed point results, Filomat, 28 (2014), 37-48.

[6] E. Karapinar, On best proximity point of psi-Geraghty contractions, Fixed Point Theory and Applications, 2013 (2013), Article number: 200, 9 pages.

[7] E. Karapinar, A discussion on alpha-psi-Geraghty contraction type mappings, Filomat, 28 (2014), 761-766.

[8] M. Geraghty, On contractive mappings, Proceedings of the American Mathematical Society, 40 (1973), 604-608.

[9] A. Meir, E. Keeler, A theorem on contraction mappings, Journal of Mathematical Analysis and Applications, 28 (1969), 326-329.

[10] T. Suzuki, Fixed point theorem for a kind of Cirić type contractions in complete metric spaces, Advances in the Theory of Nonlinear Analysis and its Applications, 2 (1) (2018), 33-41.

\author{
ÖzLem ACAR \\ SElÇUK University \\ FACULTY OF SCIENCE \\ Department of Mathematics \\ 42003, KONYA \\ TURKEY \\ E-mail address: acarozlem@ymail.com
}

